\title{
Association of Mammographic Density Reduction with Hormone Therapy and Relapse in Asian Breast Cancer Patients: A Multivariate Survival Analysis
}

\section{Wei-Chung Shia}

Changhua Christian Medical Foundation Changhua Christian Hospital

Hwa-Koon Wu

Changhua Christian Medical Foundation Changhua Christian Hospital

\section{Li-Sheng Lin}

The Affiliated Hospital of Putain University

Dar-Ren Chen (D115045@cch.org.tw)

Changhua Christian Medical Foundation Changhua Christian Hospital https://orcid.org/0000-00030631-2518

\section{Research Article}

Keywords: mammographic density, hormone therapy, breast cancer

Posted Date: March 19th, 2021

DOl: https://doi.org/10.21203/rs.3.rs-272602/v1

License: (9) This work is licensed under a Creative Commons Attribution 4.0 International License. Read Full License 


\section{Abstract}

\section{Purpose}

Currently, the ability to predict the development of resistance to hormone therapy (HT) in breast cancer patients is suboptimal, and attempts are ongoing to develop more specific prognostic markers for endocrine resistance. Several studies have shown that mammographic breast density is an important predictor of outcomes following adjuvant HT. This study aimed to evaluate HT-induced mammographic density (MD) reduction (MDR) and its association with relapse and metastasis in Taiwanese patients.

\section{Methods}

In this retrospective study conducted at the Changhua Christian Hospital, 1553 breast cancer patients were screened. Of these, 399 with luminal-like cancer who were first diagnosed between January 2011 and December 2017 and had received adjuvant HT (include treatment with tamoxifen, aromatase inhibitor or mixture) were enrolled. The mean follow-up period for all patients was 1847 days (median follow-up period was 1815 days). The MD was measured as a percentage using the automatic volumetric mammographic density estimation.

\section{Results}

We found the MDR equally $20.8 \%$ which MD measured was before and after surgery and HT during 6 to 18 months was a significant cut-off threshold for good or pool diagnosis in breast cancer patients who receiving $\mathrm{HT}$. By utilized the Cox proportion-hazards model analysis, the disease-free survival rate was significantly higher in MDR-positive patients (MDR > 20.8\%) than in MDR-negative patients (MDR < $20.8 \%)(p=0.048)$. Age, histologic grade, histologic stage, and lymph node metastasis showed a significant association with recurrence-free survival in all patients.

\section{Conclusions}

Our results demonstrate the feasibility of using MDR to predict the preliminary outcomes of adjuvant HT in Asian breast cancer patients.

\section{Introduction}

Adjuvant hormone therapy $(\mathrm{HT})$ is effective in inhibiting the growth of hormone-sensitive (luminal-like) tumors in breast cancers. The ability to predict the development of resistance to HT in breast cancer patients is suboptimal and attempting to develop more specific prognostic markers that can predict endocrine resistance. It has been shown that mammographic density (MD) reduction (MDR) during therapy is associated with a risk decreasing of breast cancer progression.

Currently, the mechanisms underlying MDR are not completely understood. Previous studies have shown that tamoxifen treatments are associated with a reduction in breast density [1, 2]. However, this finding is 
inconsistent in aromatase inhibitor (Al) treatment, according to several previous studies. The effect of Als to breast density was from no effect [3] to average 5.9\% decreasing [2]. Even it is having the divergent views of this issue, a study reveals the overall aromatase expression is higher in tissue cores taken from dense regions than in those from non-dense regions of the breasts of healthy women [4] and cannot ruleout the possibility of influence of Als treatment to breast density. However, it did not completely support a significant MDR when compared to control groups in both small $[5,6]$ and large cohorts [3] in postmenopausal women treated with Als.

There is no clear definition of a clinically meaningful MDR threshold up till the present moment due to the heterogeneity between studies, include (1) cohort size (2) population (3) focus on tamoxifen or Als treatment or (4) if consideration of body mass index (BMI) as a factor. Therefore. it is interesting issue to investigating the potential of MDR as a potential indicator of response to hormonal manipulations. This study aimed to evaluate the feasibility of using MDR as a response predictor of adjuvant HT in Asian breast cancer patients by combining the group effect of tamoxifen or Als on MD totally.

\section{Methods}

\section{Study}

This retrospective study was based on the existing medical records of patients with luminal-like breast cancer in the breast cancer registry and raw mammographic data at the Changhua Christian Hospital from 2011 to 2015. A total of 1941 ER-positive patients were screened. The participants were aged 35-75 years and having full record of mammographic exam during whole adjuvant $\mathrm{HT}$ treatment. The patient's age, calculated BMI (in $\mathrm{kg} / \mathrm{m}^{2}$ ), tumor size, grade, stage, lymph node status, estrogen receptor (ER)/progesterone receptor (PR)/human epidermal growth factor receptor 2 (HER2) status, and MD grade before HT were obtained for all participants from their medical records. The exclusion criteria include (a) non-luminal-like patient (ER negative), (b) not administrated with HT after confirmed diagnosis, (c) had bilateral breast or occult breast cancer or any other condition which was not appropriate for evaluation, and (d) underwent treatment for less than 48 months. To prevent over express the treatment effect of tamoxifen on MDR, patients with T0/Tis/T1 stage disease in TNM classification were also excluded. Treatment with adjuvant HT was generally decided according to the institution's guidelines. The standard duration of treatment with tamoxifen is 5 years. Postmenopausal women were treated with the aromatase inhibitors anastrozole and letrozole for up to 5 years after surgery or after 2 to 3 years of tamoxifen. A detailed flow of patient selection is shown in Figure 1.

\section{MD measurement}

In this study, the MD measurement was utilized the full-field digital mammography (FFDM) combining automated mammographic density estimated software. It minimizes the bias, compared to traditional human visual assessment. All participants were undergoing the same procedure of institute and had four- 
view digital mammograms in each exam. Standard two-dimensional digital mammograms were obtained for all the participants using mainstream FFDM systems, including Senographe Essential/Senographe DS (GE Medical Systems, Milwaukee, WI, USA), Mammomat Inspiration (Siemens AG Healthcare, Erlangen, Germany), and Selenia Dimensions systems (Hologic Inc, Bedford, MA, USA). The MD measurements, including the mammographic density grade (VDG), overall dense breast volume (in $\mathrm{cm}^{3}$ ), and volumetric density percentage (VPD), were estimated and quantitated using the Volpara ${ }^{\mathrm{TM}}$ software version 1.5.1 (Volpara Health Technologies, Wellington, New Zealand). To decrease the effect of tumor tissues on the density estimates and to obtain more accurate results, the VPD in the present study were calculated only from the contralateral lesions. The change in the MD at the last measurement compared to that at baseline was used to calculate the MDR. It is note that the VDG in VolparaTM have different density grade estimation method to the Breast Imaging Reporting and Data System (BI-RADS). However, it is proved the performance and accuracy of density grade estimation was equal to experienced radiologist. We do not repeat the mammographic breast density measurement of consistent between radiology and VDG again.

\section{MDR measurement}

Mammographic density reduction (MDR) was based on two digital mammograms; the first was the baseline mammogram which taken before surgery and adjuvant HT (preMD), and the second was 6 to 18 months after the start of adjuvant HT (postMD). It defined as the absolute MDR of these two images:

$\operatorname{MDR}(\%)=$ preMD - postMD $/ 100$

The diagnostic accuracy of MDR threshold was estimated using the area under the receiver operating characteristics (ROC) curve (AUC) and was compared with DeLong's nonparametric test. The Youden's index [7] was utilized to determine the optimal cut-off point for the specificity and sensitivity result.

\section{Statistics analysis}

Cox proportional hazards model was used to estimate the disease-free survival (DFS) rate and survival curve. We also identified the different factors that are associated with MDR on DFS and hazard ratios (HRs) to represent the risks through Cox proportional hazards model. The overall mode fit estimation in Cox regression model was utilized the Chi-squared statistic tests the relationship between time and all the covariates in the model, to ensure the proportional hazard assumption is satisfied. The p-value less than 0.05 was considered significant in statistic and in model fit. Thee statistical analyses were performed using MedCalc for Windows (Version 19.5.3, MedCalc Software, Ostend, Belgium).

\section{Results}

\section{Patient characteristics}


After screening the breast cancer registration database, 399 patients with luminal-like breast cancer who were first diagnosed between 2011 to 2017 and had received adjuvant HT were recruited. Of these 23 patients $(18.1 \%)$ had ductal carcinoma in situ (DCIS). The mean age of the enrolled patients was 53.16 years $(S D=11.44$ years). Each patient had complete mammographic records for the whole duration of the follow-up, and all follow-up days was converted to years for the time scale in KM curve analysis and Cox regression in this study. The average follow-up was for 5.11 years $(S D=1.64)$. The follow-up period from the start of adjuvant $\mathrm{HT}$ to the last mammogram was comparable in MDR-positive and -negative patients ( 5.2 years \pm 1.55 vs. 5.09 years \pm 1.67$)$. There are 50 patients $(12.53 \%)$ had tumor recurrence or metastasis during the follow-up. Having the extremely dense breasts (mammographic density grade equally to ' $d$ ', according to BI-RADS $5^{\text {th }}$ criteria) prior to therapy (initial breast density) were noted in 165 patients (41.35\%). Table 1 presents the summarize of the participants details.

\section{Threshold for MDR-positive}

The cut-off of MDR in $20.8 \%$ was found having the optimal sensitive and specificity in metastatic / relapse association. By utilized the ROC curve estimation, the sensitive was $80.0 \%$ and the specificity is $80.5 \%$ (AUC $=81.2 \%$ ) in the accuracy of a diagnostic test. Figure 2 shows the ROC curves and the AUC for MDR-positive group when cut-off equally $20.8 \%$. Figure 3 presents the DFS rates of the MDR-positive (MDR > 20.8\%) and MDR-negative (MDR < 20.8\%) patients in Kaplan-Meier curves ( $p=0.041$ in log-rank test).

\section{Factors associated with MDR}

We evaluated the significance of each factor from patients which may associated with MDR by utilizing multivariate logistic regression analysis. These factors were including age, BMI (calculated in $\mathrm{kg} / \mathrm{m}^{2}$ ), tumor size (in continuous), lymph node metastasis status, pathologic stage, histologic grade, ki-67 positive status ( $>10 \%$ at labeling), HER2 positive status and menopausal status (Age $>50$ years). The analysis result was shown in table 2 , and the age $\left(p=2 \times 10^{-3}\right)$ was the only significant factors after the test.

\section{MDR and recurrence-free survival}

Table 3 summarizes the effects of various factors on the association between MDR and recurrence-free survival of whole study group. In the multivariate analyses by Cox proportional hazard model, MDR positivity remained an independent predictor of total recurrence-free survival $(H R=0.881 ; p=0.032)$ after adjusting for several prognostic factors (age, BMI, tumor size, histologic grade status, pathologic stage status, menopausal status, lymph node status and Ki-67 / HER2 status). High histologic grade (grade $\geq$ 3 ) and HER2 positivity were also significantly associated with recurrence-free survival. 
In the table 3, although the menopause status was not significant level in statistic, it is close and slightly over this threshold $(p=0.056)$. Separate analysis of premenopausal women and postmenopausal women may help to find more related factors. The multivariate regression analysis for the postmenopause women group was also provided here. Table 4 summarizes the findings of the multivariate Cox proportional hazard model analyses in the post-menopausal group (Age $>50$ years) after adjusting for several prognostic factors. MDR positivity was also a significant predictor of recurrence in this group $(p=0.038)$. High histologic grade $(p=0.004)$, tumor size $>2 \mathrm{~cm}(p=0.031)$ and HER2 positive $(p<0.001)$ were all significantly associated with recurrence-free survival in the post-menopausal group. An interesting finding is the factor tumor size $>2 \mathrm{~cm}$ was the significant factor with recurrence-free survival in postmenopausal women only and not in whole study group.

\section{Discussion}

This study assesses the changes in mammographic breast density in Taiwanese breast cancer patients, following HT and its association with relapse. First, we have found MDR threshold and confirmed that MDR positivity (MDR $>20.8 \%$ ) is an independent prognostic factor of recurrence-free survival based on Kaplan-Meier survival curves. Second, we have identified the factors associated with MDR positivity and recurrence-free survival by utilized multivariate logistic regression analysis. Logistic regression analysis showed that age (continuous variable), tumor size $(>2 \mathrm{~cm}$ ), high pathologic stage (Stage $\geq 3$ ) were significant factors related to MDR status. In the analysis of MDR-positive patients, high histological grade (grade $\geq 3$ ) or high pathologic stage (stage $\geq 3$ ) and lymph node metastasis were significantly associated with recurrence.

Several population-based related studies which including Caucasian and Korea has demonstrated that the MD grade before HT, menopause, and HER2 status are associated with MDR [8]. In addition to the HER2 status, the other factors were not found in this study. For these inconsistent findings, possibly reasons including the differences in physiological mechanisms of the study population as well as the different methods used to evaluate breast density. For example, the Caucasian women usually have a higher BMI and body size [9] compared to Asian women. The trend of MD decreasing between premenopausal and post-menopausal patients after HT seems also seems to be a difference. An analysis in volumetric breast density with tamoxifen and Al treatment of Caucasian-based study [10] revealed the post-menopausal Al patient had greatest reduction of VPD, and this result is inconsistent to the analysis of our study cohort (mean VPD decline of post-menopausal Al patients: $0.3 \%$, post-menopausal tamoxifen patients: $1.5 \%$ ). In the meantime, a previous review article [11] also indicated the MD response is not a good biomarker in predicting Al effect of breast cancer patient in White women. Although the association of DFS rate of Al treatment Asian breast cancer patients and MDR is not the focus of this article, the proportion of post-menopausal Al treatment patients in this study group was $72.27 \%$ (full Al treatment $=44.55 \%$, combine Al and tamoxifen $=27.72 \%$, and the Cox proportion hazard model still presented the MDR-positive is an association factor to DFS and decrease the hazard of relapse (HR = 0.903 and $p=0.045$, detailed shown in Table 4), even though the $p$-value has already close to the upper bound of significant level of statistic. 
Previous studies based on Caucasian women that have indicated a 10\% threshold of MDR as a cut-off to reduce the risk of recurrence in breast cancer $[8,12]$. An issue is this finding should be validated in Asian women due to having different distribution of mammographic density. In our analysis, the MDR cut-off was $20.8 \%$ and found to be statistically significant between MDR-positive and negative in the KaplanMeier recurrence-free survival curves. This threshold was higher than previous finding. Since of the different distribution of breast volume and density between Asian and Caucasian women was reported [13], it possible the reason of this different. It also demonstrates the association between MD decreasing in specific proportion during HT and better diagnosis may not be influenced by the difference in populations. In addition, the MD measurement in previous studies was based on computer-aid quantitative assessment (semi-automatic mammographic density measurement), it is still relying on the visual reading by radiologists. Our study utilized fully-automated volumetric mammographic MD measurements, which reduced the effort and bias compared to the previous methods.

A significant issue of this study is about 80\% participants were excluded in screening (1941 participants were screening initially and 399 were enrolled finally). It may cause the bias and patient selection issue. The reason of this is follow-up duration of the most participants were not exceed the criteria of this study. Only the participant meets the criteria require were enrolled. Because of the high medical resource denseness and accessibility in Taiwan, whole adjuvant HT treatment of patients may collaborate with two or more hospital, therefore fewer patients finished all treatment in the same institute. This issue will be improved in the future by enrolling more participants and collaborating with other institutes.

In conclusion, in addition to the significant MDR threshold in clinical need to be classify, the Asian breast cancer patient who receiving HT might have benefited from the Caucasian-based related studies.

However, how to classify the MDR threshold was not easy, and the actual threshold may variate due to different population / enrolled criteria of HT patient cohort. It is important to compared these related studies at the same or similar enrolled criteria or population. The aims of this study are finding a meaningful MDR threshold and predict the outcomes of adjuvant HT in Asian breast cancer patients, and the preliminary result showing the statistically significant. It wished to contributed this to clinical in the future after enlarge the study cohort.

\section{List Of Abbreviations}

HT

Hormone therapy; MD:Mammographic density; MDR:Mammographic density reduction; Al:Aromatase inhibitor; BMI:Body mass index; FFDM:Full-field digital mammography

\section{Declarations}

\section{Availability of data and material}


The datasets generated and/or analyzed during the current study are not publicly available due to the policy of the IRB but are available from the corresponding author on reasonable request.

\section{Ethics approval and consent to participate}

This study was approved by the Institutional Review Board of the Changhua Christian Hospital, Changhua, Taiwan (No. 171217). The requirement for informed consent of the patients was waived due to the retrospective nature of the study.

\section{Consent for publication}

Not applicable.

\section{Competing interests}

None.

\section{Availability of data and material}

The datasets generated and analyzed during the current study are not publicly available due to IRB and institutional restrictions, but are available from the corresponding author on reasonable request.

\section{Funding}

Not applicable.

\section{Authors' contributions}

WCS was involved in manuscript drafting, method design, and statistics analysis. DRC was involved in framing the main proposal of the study and in data collection. HKW was involved in acquiring the mammographic data. DRC was involved in patient recruitment. WCS and HKW were involved in the mammography analysis. DRC and HKW were involved in the adjuvant HT period data and histology data collection.

\section{Acknowledgements}

The authors would like to thank the Hung-Tin Lin of the Cancer Research Center, Sing-Yin Chen and WenTing Shen of the Molecular Medicine Laboratory, Changhua Christian Hospital for their help in collecting and checking the clinical data from the enrolled patients. The authors are also grateful to Elsevier's English language editing services for the proofreading of this article.

\section{References}

1. Cuzick J, Warwick J, Pinney E, Warren RM, Duffy SW (2004) Tamoxifen and breast density in women at increased risk of breast cancer. J Natl Cancer Inst 96(8):621-628 
2. Kim J, Han W, Moon H-G, Ahn SK, Shin H-C, You J-M, Han S-W, Im S-A, Kim T-Y, Koo HR (2012) Breast density change as a predictive surrogate for response to adjuvant endocrine therapy in hormone receptor positive breast cancer. Breast Cancer Res 14(4):R102

3. Vachon CM, Suman VJ, Brandt KR, Kosel ML, Buzdar AU, Olson JE, Wu FF, Flickinger LM, Ursin G, Elliott CR, Shepherd L, Weinshilboum RM, Goss PE, Ingle JN (2013) Mammographic breast density response to aromatase inhibition. Clin Cancer Res 19(8):2144-2153. doi:10.1158/1078-0432.CCR$12-2789$

4. Vachon CM, Sasano H, Ghosh K, Brandt KR, Watson DA, Reynolds C, Lingle WL, Goss PE, Li R, Aiyar SE, Scott CG, Pankratz VS, Santen RJ, Ingle JN (2011) Aromatase immunoreactivity is increased in mammographically dense regions of the breast. Breast cancer research treatment 125(1):243-252. doi:10.1007/s10549-010-0944-6

5. Mousa NA, Crystal P, Wolfman WL, Bedaiwy MA, Casper RF (2008) Aromatase inhibitors and mammographic breast density in postmenopausal women receiving hormone therapy. Menopause 15(5):875-884. doi:10.1097/gme.0b013e31816956c3

6. Smith J, Dilawari A, Ursin G, Andreopoulou E, Checka C, Axelrod D, Guth A, Toth H, Utate M, Carapetyan K, Reich E, Diflo T, Muggia F (2012) A pilot study of letrozole for one year in women at enhanced risk of developing breast cancer: effects on mammographic density. Anticancer research 32(4):1327-1331

7. Ruopp MD, Perkins NJ, Whitcomb BW, Schisterman EF (2008) Youden Index and optimal cut-point estimated from observations affected by a lower limit of detection. Biometrical journal Biometrische Zeitschrift 50(3):419-430. doi:10.1002/bimj.200710415

8. Ko KL, Shin IS, You JY, Jung SY, Ro J, Lee ES (2013) Adjuvant tamoxifen-induced mammographic breast density reduction as a predictor for recurrence in estrogen receptor-positive premenopausal breast cancer patients. Breast cancer research treatment 142(3):559-567. doi:10.1007/s10549-0132726-4

9. Deurenberg P, Yap M, van Staveren WA (1998) Body mass index and percent body fat: a meta analysis among different ethnic groups. International Journal Of Obesity 22:1164. doi:10.1038/sj.ijo.0800741

10. Engmann NJ, Scott CG, Jensen MR, Ma L, Brandt KR, Mahmoudzadeh AP, Malkov S, Whaley DH, Hruska CB, Wu FF (2017) Longitudinal changes in volumetric breast density with tamoxifen and aromatase inhibitors. Cancer Epidemiology Prevention Biomarkers 26(6):930-937

11. Vachon CM, Suman VJ, Brandt KR, Kosel ML, Buzdar AU, Olson JE, Wu F-F, Flickinger LM, Ursin G, Elliott CR (2013) Mammographic breast density response to aromatase inhibition. Clin Cancer Res 19(8):2144-2153

12. Cuzick J, Warwick J, Pinney E, Duffy SW, Cawthorn S, Howell A, Forbes JF, Warren RM (2011) Tamoxifen-induced reduction in mammographic density and breast cancer risk reduction: a nested case-control study. J Natl Cancer Inst 103(9):744-752. doi:10.1093/jnci/djr079 
13. Shia WC, Wu HK, Huang YL, Lin LS, Chen DR (2018) Mammographic Density Distribution of Healthy Taiwanese Women and its Naturally Decreasing Trend with Age. Scientific reports 8(1):14937. doi:10.1038/s41598-018-32923-z

\section{Tables}

Table 1. Clinicopathologic characteristics of patients

Primary Tumor Stage is listed according to the pathologic status. T stage is based on the TNM grading system. TAM: Tamoxifen, Als: Aromatase inhibitors 


\begin{tabular}{|c|c|c|c|}
\hline Associated Variable & $\begin{array}{l}\text { Total } \\
(N=399)\end{array}$ & $\begin{array}{l}\text { MDR-positive } \\
(N=103)\end{array}$ & $\begin{array}{l}\text { MDR-negative } \\
(N=296)\end{array}$ \\
\hline Mean age $($ year $\pm S D)$ & $53.16 \pm 11.44$ & $50.15 \pm 10.97$ & $54.20 \pm 11.42$ \\
\hline \multicolumn{4}{|l|}{ Menopausal } \\
\hline Pre-menopausal & 179 & 62 & 117 \\
\hline Post-menopausal & 220 & 41 & 179 \\
\hline \multicolumn{4}{|l|}{ Follow-up time } \\
\hline Mean (days $\pm S D)$ & $1841.7 \pm 591.2$ & $1871.9 \pm 557.6$ & $1830.7 \pm 602.9$ \\
\hline \multicolumn{4}{|l|}{$B M I$} \\
\hline $\operatorname{Mean}\left(\mathrm{kg} / \mathrm{m}^{2} \pm S D\right)$ & $24.57 \pm 4.27$ & $24.34 \pm 4.51$ & $24.65 \pm 4.19$ \\
\hline \multicolumn{4}{|l|}{ Hormone therapy } \\
\hline TAM only & 208 & 65 & 143 \\
\hline Als only & 105 & 17 & 88 \\
\hline TAM /Als combination & 86 & 21 & 65 \\
\hline \multicolumn{4}{|c|}{ Mammographic density grade (in VDG) } \\
\hline$b$ & 57 & 9 & 48 \\
\hline$c$ & 177 & 33 & 144 \\
\hline$d$ & 165 & 61 & 104 \\
\hline \multicolumn{4}{|l|}{ Primary tumor stage } \\
\hline$T 1(a / b / c)$ & 201 & 57 & 144 \\
\hline$T 2$ & 179 & 41 & 138 \\
\hline$T 3 / T 4(a / b / c)$ & 19 & 5 & 14 \\
\hline \multicolumn{4}{|l|}{ Lymph node status } \\
\hline Negative & 237 & 67 & 170 \\
\hline Positive & 53 & 32 & 121 \\
\hline \multicolumn{4}{|l|}{ HER2 } \\
\hline Negative & 186 & 45 & 141 \\
\hline
\end{tabular}




\begin{tabular}{|c|c|c|c|}
\hline Positive & 213 & 58 & 155 \\
\hline \multicolumn{4}{|l|}{$K i-67$} \\
\hline \multicolumn{4}{|l|}{ High } \\
\hline \multicolumn{4}{|l|}{ Low } \\
\hline \multicolumn{4}{|c|}{ Histologic grade } \\
\hline $1 / 2$ & 356 & 91 & 265 \\
\hline 3 & 40 & 11 & 29 \\
\hline \multicolumn{4}{|c|}{ Metastasis } \\
\hline$Y$ & 35 & 11 & 24 \\
\hline$N$ & 364 & 92 & 272 \\
\hline \multicolumn{4}{|c|}{ Recurrence } \\
\hline$Y$ & 23 & 2 & 11 \\
\hline$N$ & 386 & 101 & 285 \\
\hline
\end{tabular}

Table 2. Factors associated with the MDR in multivariate logistic regression analysis

*: Significant in statistics $(p<0.05),{ }^{* *}:$ Significant in statistics $(p<0.01)$ 


\begin{tabular}{|c|c|c|}
\hline Variable & Odds ratio (95\% CI) & $P$ value \\
\hline Age, years (continuous) & $0.968(0.947,0.988)$ & $0.002^{*}$ \\
\hline BMI, kg/m² (continuous) & $0.983(0.931,1.038)$ & 0.529 \\
\hline \multicolumn{3}{|l|}{ Tumor Size } \\
\hline$\leq 2 \mathrm{~cm}$ & 1.000 (Reference) & \\
\hline$>2 \mathrm{~cm}$ & $0.753(0.483,1.181)$ & 0.217 \\
\hline \multicolumn{3}{|l|}{ Lymph Node } \\
\hline Negative & 1.000 (Reference) & \\
\hline Positive & $0.806(0.387,1.678)$ & 0.56 \\
\hline \multicolumn{3}{|l|}{ Pathologic Stage } \\
\hline Low (0/1) & 1.000 (Reference) & \\
\hline $\operatorname{High}(2 / 3)$ & $0.650(0.314,1.346)$ & 0.25 \\
\hline \multicolumn{3}{|l|}{ Ki-67 } \\
\hline Negative & 1.000 (Reference) & \\
\hline Positive & $1.502(0.614,3.676)$ & 0.37 \\
\hline \multicolumn{3}{|l|}{ HER2 } \\
\hline Negative & 1.000 (Reference) & \\
\hline Positive & $0.412(0.112,1.506)$ & 0.18 \\
\hline \multicolumn{3}{|l|}{ Histologic Grade } \\
\hline Low (1/2) & 1.000 (Reference) & \\
\hline High (3) & $0.777(0.328,1.844)$ & 0.57 \\
\hline \multicolumn{3}{|l|}{ Menopausal Status } \\
\hline No & 1.000 (Reference) & \\
\hline Yes & $0.639(0.302,1.351)$ & 0.24 \\
\hline
\end{tabular}

Table 3. Effect of mammographic density reduction (MDR) on disease-free survival ${ }^{*} p<0.05,{ }^{* *} p<0.01$ 


\begin{tabular}{|lll|}
\hline Variable & Hazard Ratio $(95 \%$ Cl) & P Value \\
\hline MDR positive & $0.881(0.400-1.938)$ & $0.032^{*}$ \\
\hline Age, years (continuous) & $1.008(0.979-1.037)$ & 0.582 \\
\hline BMl, kg/m2 (continuous) & $0.996(0.918-1.081)$ & 0.926 \\
\hline High histologic grade (Grade $>3)$ & $2.136(1.101-4.514)$ & $0.047^{*}$ \\
\hline High pathologic stage $($ Stage $>3)$ & $0.667(0.121-3.673)$ & 0.642 \\
\hline Tumor size $>2$ cm & $5.039(0.918-27.678)$ & 0.063 \\
\hline Menopausal & $1.153(0.374-3.556)$ & 0.056 \\
\hline Lymph node metastasis & $2.108(0.885-5.019)$ & 0.092 \\
\hline Ki-67 positive & $0.989(0.435-2.252)$ & 0.981 \\
\hline HER2 positive & $2.983(1.413-6.299)$ & $0.004^{\star *}$ \\
\hline
\end{tabular}

Table 4. Effect of mammographic density reduction (MDR) on disease-free survival in post-menopausal patients

${ }^{*} p<0.05,{ }^{* *} p<0.01$

\begin{tabular}{|lll|}
\hline Variable & Hazard Ratio $(95 \%$ Cl) & P Value \\
\hline MDR positive & $0.903(0.335-2.435)$ & $0.045^{\star}$ \\
\hline Age, years (continuous) & $1.004(0.960-1.051)$ & 0.856 \\
\hline BMl, kg/m2 (continuous) & $0.981(0.895-1.075)$ & 0.675 \\
\hline High histologic grade (Grade >3) & $1.680(0.667-4.225)$ & $0.027^{\star}$ \\
\hline High pathologic Stage (Stage $>3)$ & $0.29(0.022-3.957)$ & 0.357 \\
\hline Tumor size $>2$ cm & $10.158(0.736-139.730)$ & $0.031^{\star}$ \\
\hline Lymph node metastasis & $2.181(0.795-5.987)$ & 0.13 \\
\hline Ki-67 positive & $0.89(0.356-2.228)$ & 0.801 \\
\hline HER2 positive & $4.233(1.758-10.192)$ & $0.001^{* *}$ \\
\hline
\end{tabular}

\section{Figures}




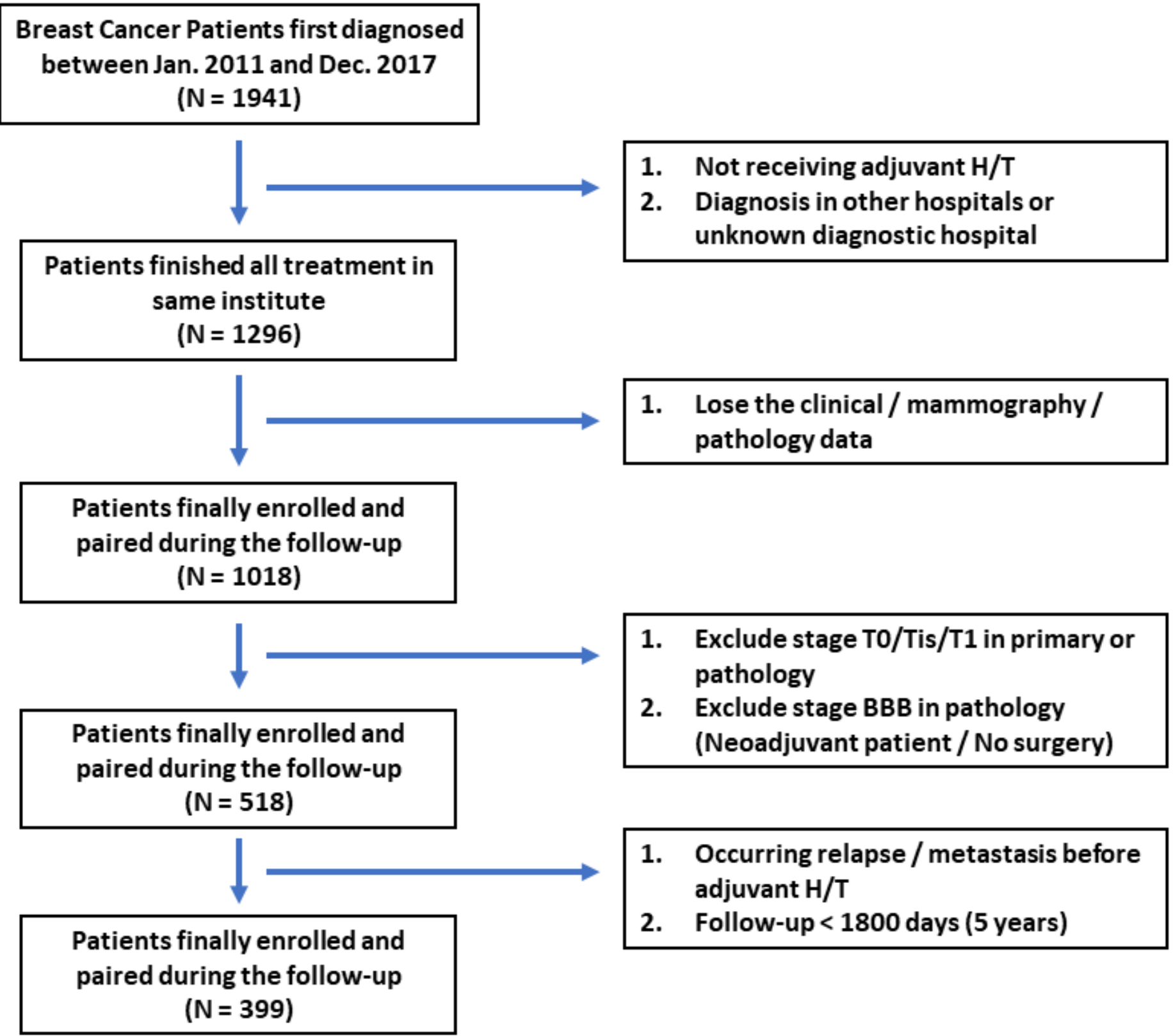

Figure 1

Flowchart of patient enrollment 


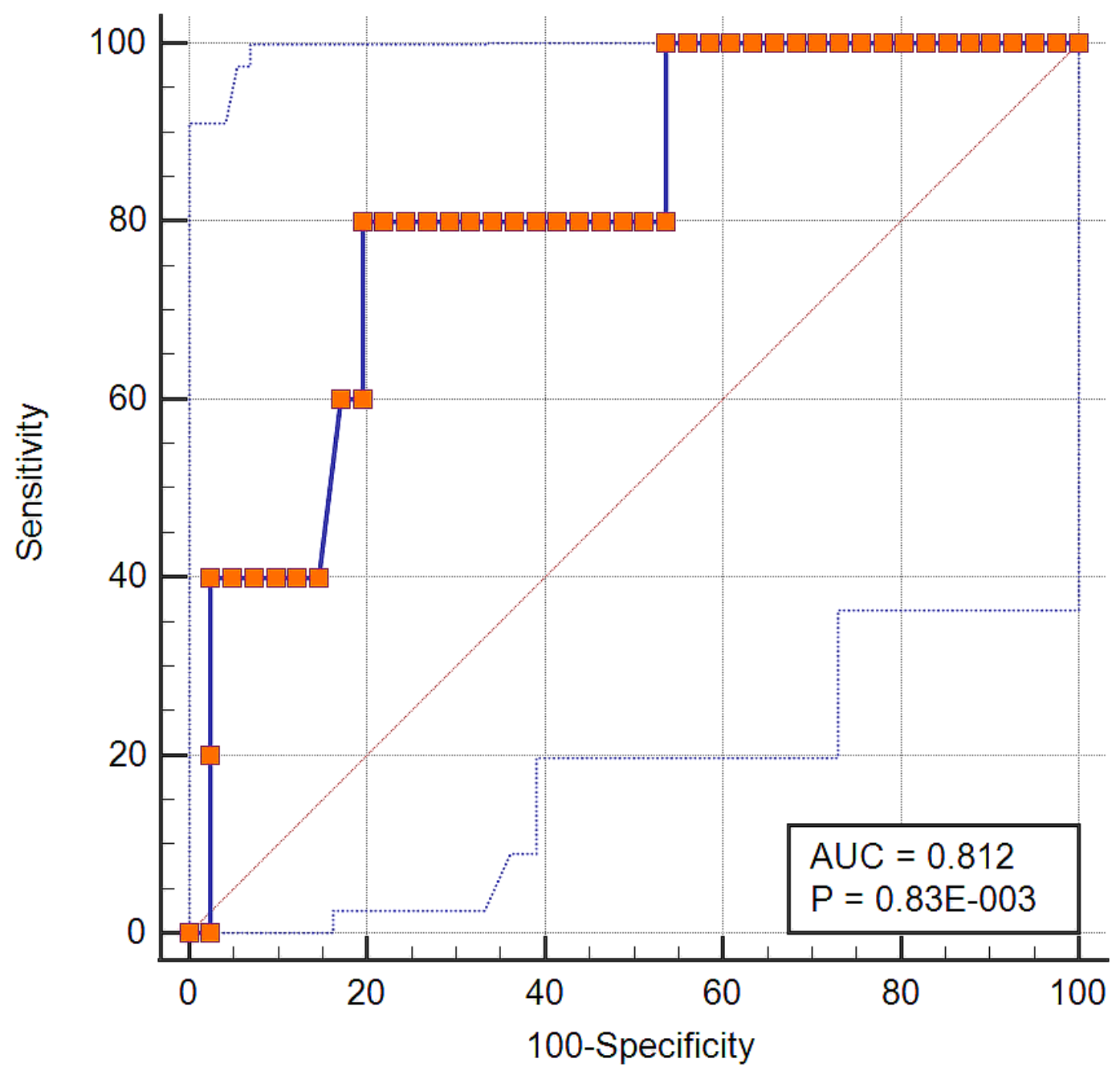

Figure 2

The ROC curve for the criteria when MDR threshold is $20.8 \%$ 


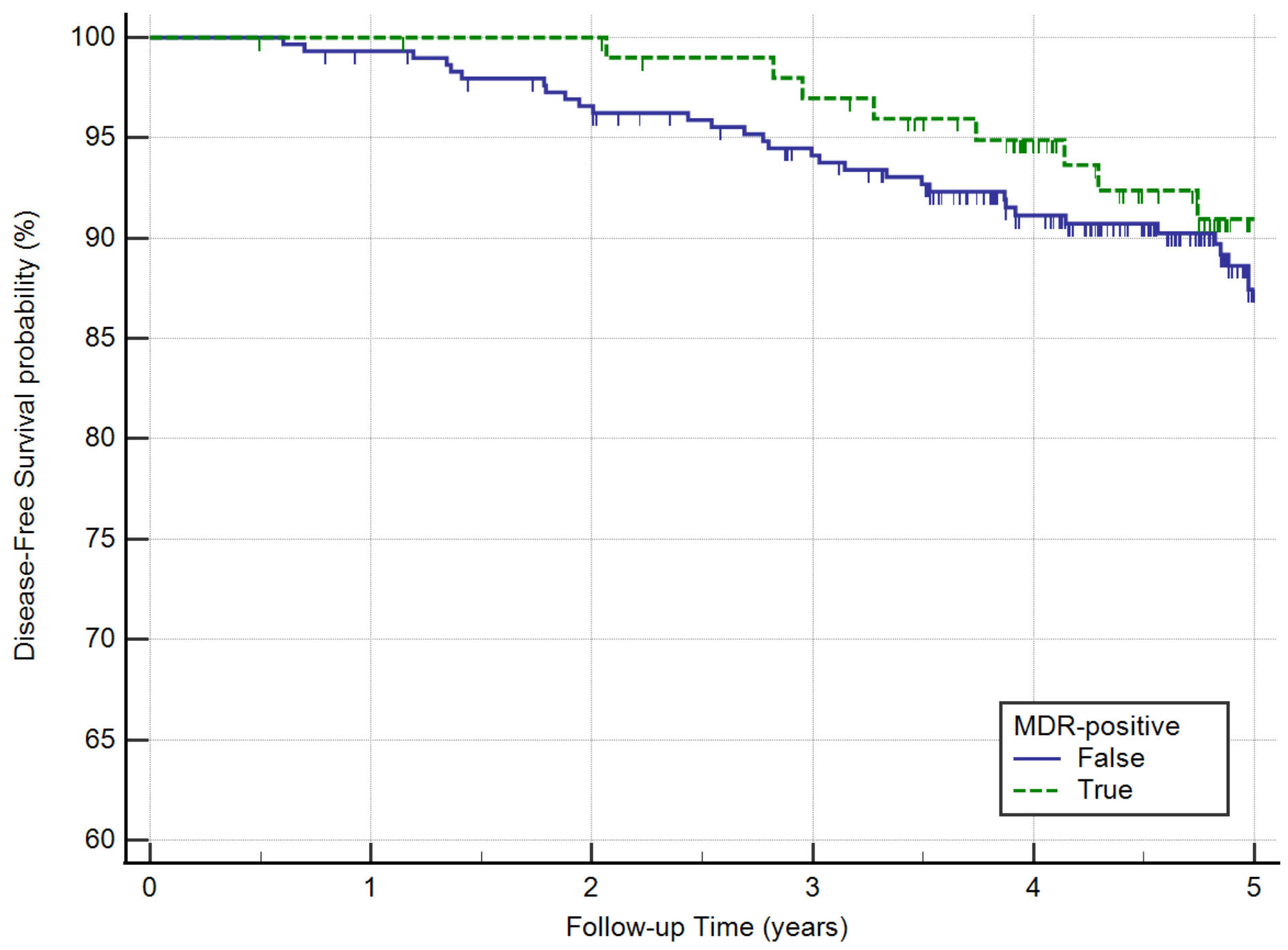

Figure 3

Cumulative survival curves for breast cancer with and without MDR 